\title{
Spinocerebellar ataxias in Venezuela: genetic epidemiology and their most likely ethnic descent
}

\author{
Irene Paradisi, Vassiliki Ikonomu and Sergio Arias
}

Dominantly inherited ataxias (spinocerebellar ataxias, SCAs) are a genetically heterogeneous group of neurologic diseases characterized by progressive cerebellar and spinal tract degeneration with ataxia and other signs, common to all known subtypes. Several types are relatively frequent worldwide, but in several countries, one specific SCA may show a higher prevalence owing to founder phenomena. In Venezuela, genetic epidemiological features of SCAs have been assessed during the last 30 years; mutations in ATXN1 (SCA1), ATXN2 (SCA2), ATXN3 (SCA3), CACNA1A (SCA6), ATXN7 (SCA7), ATXN8 (SCA8), ATXN10 (SCA10), TBP (SCA17) and ATN1 (dentatorubral pallidoluysian atrophy, DRPLA) loci were searched among 115 independent families. SCA7 was the most frequent subtype (26.6\%), followed by SCA3 (25.0\%), SCA2 (21.9\%), SCA1 (17.2\%), SCA10 $(4.7 \%)$ and DRPLA (3.1\%); in $43 \%$ of the families, the subtype remained unidentified. SCA7 mutations displayed strong geographic aggregation in two independent founder foci, and SCA1 showed a very remote founder effect for a subset of families. SCA10 families were scattered across the country, but all had an identical in-phase haplotype carried also by Mexican, Brazilian and Sioux patients, supporting a very old common Amerindian origin. Prevalence for dominant SCAs in Venezuela was estimated as 1:25000 nuclear families, provenances of which are either Caucasoid, African or Amerindian.

Journal of Human Genetics (2016) 61, 215-222; doi:10.1038/jhg.2015.131; published online 5 November 2015

\section{INTRODUCTION}

Spinocerebellar ataxias (SCAs) are a group of dominantly inherited neurodegenerative disorders characterized by progressive ataxia and dysarthria, frequently owing to dysfunction of the cerebellum and its associated systems. ${ }^{1}$ To date, 36 subtypes have been described worldwide, all with phenotypic overlap between them. The mutated gene is known in 20 subtypes, ${ }^{2}$ of which 12 are caused by trinucleotide, pentanucleotide (SCA10 and SCA31) or hexanucleotide (SCA36) expansions over the normal range. SCA1, SCA2, SCA3, SCA6, SCA7, SCA12, SCA17 and dentatorubral pallidoluysian ataxia (DRPLA) are referred to as polyglutamine ataxias, because a CAG repeat expansion occurs in a coding region of the gene. Age of onset in these ataxias is variable, but generally, it is inversely correlated with the number of repeats (CAG or otherwise).

Worldwide prevalence is quoted as 1-5:100 000 inhabitants, SCA3 reputed to be the most frequent in many populations. ${ }^{1,3}$ Nevertheless, different specific regional frequencies are common, as in southeastern Cuba, with a SCA2 frequency of $41: 100000 ;{ }^{4}$ Sweden with SCA7 as the most common form; ${ }^{5}$ SCA10 found exclusively in populations originated from Amerindians of South and North America (Brazil, Mexico); ${ }^{6,7}$ and SCA36 which occurs with high frequency in Costa da Morte (Galicia, Spain) and in western Japan (Okayama).$^{8-10}$ In those populations, a founder effect has been proven, which contributes to explain the high frequency of a particular subtype of ataxia.
The genetic epidemiological features of dominant ataxias have been ascertained in Venezuela for more than the last two decades; the frequency distribution of subtypes showed SCA7 to be the most prevalent in the country, followed by SCA3 and SCA2. This high prevalence of SCA7 is unusual in most populations. None of the 10 different studied subtypes among 115 independent families showed geographic aggregation/founder effect, except for SCA7 and SCA1. Of the 115 families, $2.6 \%$ had the infrequent SCA10, which is consistent with the expected Amerindian ethnic contribution to the Venezuelan populations.

\section{MATERIALS AND METHODS}

Sample

A total of 115 independent families with a presumptive diagnosis of hereditary dominant ataxia were studied for SCA1, SCA2, SCA3, SCA6, SCA7, SCA8, SCA10, SCA17 and DRPLA genotypes. They were referred during the last three decades to the Human Genetics Laboratory at the Venezuelan Institute for Scientific Research (IVIC) for both a molecular diagnostic search and for genetic counseling.

Precise places of birth of remote ancestors (grandparents and great grandparents) were established in each family; a family history for phenotype and age of onset of symptoms was recorded. A 5-ml blood sample was withdrawn, EDTA anticoagulated, and the DNA was extracted by a saline method as usual.

Voluntary informed consent was obtained from all family members, according to the institutional ethical guidelines. 


\section{DNA analyses}

Ataxia subtypes to be tested were chosen according to their prevalence in most worldwide populations and the ethnic composition of our admixed populations (Caucasoid, Amerindian and Sub-Saharan Africans).

Primers and PCR conditions for SCA2, SCA3, SCA17 and DRPLA were those previously described, ${ }^{11}$ as well as the primers for SCA1, ${ }^{12} \mathrm{SCA},{ }^{13}$ SCA $7,{ }^{14}$ SCA $8{ }^{15}$ and SCA $10 .{ }^{16}$ PCR-amplified products were resolved through polyacrylamide electrophoresis, using silver nitrate staining, as usual. ${ }^{17} \mathrm{Number}$ of CAG repeats at SCA1 was estimated as (bp-28)/3, for SCA2 as (bp-103)/3, for SCA3 as $(b p-160) / 3$, for SCA6 as $(b p-102) / 3$, for SCA7 as $(b p-278) / 3$, for SCA8 as (bp-259)/3, for DRPLA as (bp-96)/3, for SCA17 as (bp-131)/3 and for SCA10 pentanucleotide repeats, as (bp-127)/5. The actual number of repeats in each locus was confirmed in PCR products by sequencing at Macrogen, Seoul, Korea.

Detection of large expansions was accomplished through the repeat-primed PCR technique in SCA2, SCA7 and SCA10, according to Cagnoli et al. ${ }^{18,19}$ For SCA3, the locus-specific fluorescent-labeled primer sca3-RP 5'-FAMTTGTGATGAAAATACCTACCTCTT was designed using Oligo Explorer software (http://www.genelink.com/tools/gl-oe.asp); reverse primer sca3-RP-r $5^{\prime}$-TACGCATCCCAGTTTGAGACG(TGC) 5 was the usual ${ }^{20}$ and the flag primer (5'-TACGCATCCCAGTTTGAGACG) was the same used for the other loci. ${ }^{18}$ Analysis of the fluorescent PCR products was performed using an Applied Biosystem $3130 \mathrm{XL}$ automatic sequencer on a 50-cm capillary array with POP7 polymer, ROX-GS500 internal standard marker, filter D, injection voltage: $1.6 \mathrm{kV}$, injection time: $15 \mathrm{~s}$, run voltage: $15 \mathrm{kV}$ and run time: $1800 \mathrm{~s}$. Data were examined using the GeneMapper version 3.2 software (www.appliedbiosystems.com).

\section{Haplotype analysis}

Intragenic markers were selected for constructing in-phase haplotypes to ascertain remote ancestral relationships in SCA1, SCA7 and SCA10 families.

For ATXN1 locus, three intragenic polymorphisms were selected: rs140803 (intron 2, -/insTTTAA), rs179971 (intron 7, G>A) and rs180015 (intron 8, $\mathrm{C}>\mathrm{G}$, reverse orientation) and primers were designed with Primer3 software at http://www.ncbi.nlm.nih.gov/tools/primer-blast/:

ATXN1rs140803 f 5'-CAATGATGAAAGTGCACAGCTA

ATXN1rs140803 r 5'-ATCGAGTCCTCAACTTCTTCC

ATXN1rs179971 f 5'-TCTCTCTGCCCAACATCCCTA

ATXN1rs179971 r 5'-TCAGAGAAAGGAGCCACGTT

ATXN1rs180015 f 5'-TGGGTGCTCTGTCATTACTGG

ATXN1rs180015 r 5'-AGGAAGAAGGAAGGGAACAAATGA

Genotypes at each marker were established by the restriction enzymes RsaI and AciI for the single nucleotide polymorphisms (SNPs), respectively, and by electrophoretic migration for the INDEL.

For ATXN7 locus, three intragenic INDELs were used: rs144754108 (insA; intron 1), rs3841968 (delTG; intron 5) and rs3836530 (delT; 3'UTR). Primers

ATXN7rs4108 f 5'-CCTCAGAGTAAAACGCGTGC

ATXN7rs4108 r 5'-TGTGTGTGGTAGAGGTGTCT

ATXN7rs1968 f 5'-ACCTTAATGTTTCTCTTTTATGCAG

ATXN7rs1968 r 5'-CAGCAGATGATAAAACCAGC

ATXN7rs6530 f 5'-AGAAGAAAGAAAATGCAAGCTAGT

ATXN7rs6530 r 5'-AGCAGAAATCTACACTTAGTCCCA

were designed as before. Genotypes at each marker were established by sequencing at Macrogen, in index cases and in relatives.

For ATXN10 locus, previously reported ${ }^{21}$ SNPs were used: rs5764850 $(A>C), \quad$ rs72556348 (G>A, SNP_1), $\quad$ rs72556349 (G>A, SNP_2), rs72556350 (C>T, SNP_3) and rs136005 (C>T). Genotypes were established by sequencing, as mentioned.

Amerindian allele 9 of the D9S1120 microsatellite marker was assessed as previously reported. ${ }^{11}$

\section{Prevalence}

Prevalence figure during the 1990-2015 period was estimated as previously reported. ${ }^{22}$

\section{RESULTS}

In 64 out of 115 (56\%) independent families, the mutated locus was identified; in 51 index cases (44\%), the SCA subtype remained unidentified, because none of the ascertained loci had expanded alleles.

Among identified subtypes, SCA7 was the most frequent $(26.6 \%)$ with 17 independent families, followed by SCA3 (16 independent families, $25.0 \%$ ), SCA2 (14 independent families, $21.9 \%$ ), SCA1 (11 independent families, 17.2\%), SCA10 (3 independent families, $4.7 \%$ ) and DRPLA (2 independent families, 3.1\%). None of the studied families had either SCA6, SCA8 or SCA17.

Table 1 shows the main clinical manifestations in patients, at diagnosis. As expected, almost all signs were common in all subtypes, but the frequencies of some clinical features varied between them. For instance, nystagmus was very frequent in SCA3 but not in SCA1 or SCA7, and hyperreflexia was very frequent in SCA1 and SCA7, but not in SCA3. Some signs were almost exclusive of a subtype, as the visual impairment in SCA7 and seizures in SCA10.

\section{SCA1}

Normal CAG repeats range varied between 27 and 33, 31 being the most frequent allele (25.0\%), followed by $32(18.8 \%)$ and $33(15.6 \%)$; 27 and 28 CAG alleles had frequencies of $12.5 \%$ each.

A total of 199 individuals at risk of being SCA1 carriers (symptomatic and asymptomatic) were assessed for expanded alleles at the ATXN1 locus (6p22.3), of which 26 had expansions, with allele sizes between 39 and 63 CAG. Allele size instability was narrow, between -2 to $+8 \mathrm{CAG}$, maternal transmissions being more stable (range -1 to $+2 \mathrm{CAG}$ ) with $57 \%$ of alleles remaining unchanged ( $n=7$ maternal meiosis); the paternal transmissions range $(n=3)$ varied between -2 and +8 CAG. Disease onset occurred frequently after the fourth decade of life (mean age $=41.6$ years (range $27-46$ years, mode $=42$ ), $n=15$ patients with known age of onset) and there was a moderately high correlation between allele size and age of onset, with a $\mathrm{R}^{2}=0.5826(\mathrm{y}=-0.0227 \mathrm{x}+2.6636$ for log-transformed age of onset, $n=15$ observations). The most frequent initial symptom was loss of equilibrium $(60 \%)$.

Nine out of 11 families had Venezuelan remote ancestors, and they did not show geographic aggregation into a restricted focus $(55 \mathrm{~km}$ diameter), although 5 of them came from oriental states in the country: Monagas, Anzoátegui and Bolívar (Figure 1). Two of the families coming from Monagas (La Inglesa) had a recent common ancestor. All these five families plus one family coming from Miranda State (El Café, $335 \mathrm{~km}$ far apart) shared the same in-phase haplotype insTTTAA; $\mathrm{G} ; \mathrm{C}$; this haplotype was absent in control chromosomes $(n=32)$. The remaining families with heterogeneous origins had either delTTTAA;A;G, insTTTAA;A;G or insTTTAA;A;C in-phase haplotypes, with the same frequencies that control chromosomes $(12.5 \%$ each). Interestingly, the most frequent haplotype delTTTAA;A;C in control chromosomes $(45.8 \%)$ was not in-phase with ATXN1 mutations.

\section{SCA2}

Forty-four out of 199 studied individuals belonging to 14 independent families had expanded alleles between 39 and 67 CAG repeats at the ATXN2 locus (12q24.12). Meiotic instability of the mutated allele was observed in both maternal and paternal transmissions; the range of variation in maternal meiosis was -3 to +20 CAG $(n=19)$ and in the paternal ones, it was -2 to +27 CAG $(n=7)$. In three independent instances, large expansions of the parent-mutated allele led to a disease with an infantile onset: a father carrying a 40 CAG repeat allele transmitted a 67 CAG allele to his daughter, who had a disease onset at 
Table 1 Frequency of main clinical phenotypes at diagnosis in affected subjects, according to ataxia subtype

\begin{tabular}{|c|c|c|c|c|c|c|}
\hline \multirow[b]{3}{*}{ Clinical features } & \multicolumn{6}{|c|}{ Ataxia subtypes } \\
\hline & SCA1 & SCA2 & SCA3 & SCA7 & SCA10 & DRPLA \\
\hline & $\mathrm{n}=15$ & $\mathrm{n}=18$ & $\mathrm{n}=16$ & $n=25$ & $\mathrm{n}=6$ & $n=2$ \\
\hline Ataxia & $100 \%$ & $100 \%$ & $100 \%$ & $100 \%$ & $100 \%$ & 0 \\
\hline Dysarthria & $100 \%$ & $100 \%$ & $100 \%$ & $100 \%$ & $100 \%$ & 0 \\
\hline \multicolumn{6}{|l|}{ dysdiadochokinesia } & $50 \%$ \\
\hline Romberg test (+) & $27 \%$ & $50 \%$ & $63 \%$ & $48 \%$ & 0 & 0 \\
\hline Visual impairment ${ }^{a}$ & $7 \%$ & $6 \%$ & 0 & $100 \%$ & 0 & 0 \\
\hline Diplopia & $7 \%$ & 0 & $13 \%$ & $16 \%$ & 0 & 0 \\
\hline Ocular apraxia & $7 \%$ & $11 \%$ & $13 \%$ & $8 \%$ & $17 \%$ & 0 \\
\hline Nystagmus & $20 \%$ & 0 & $81 \%$ & $20 \%$ & $33 \%$ & 0 \\
\hline Seizures & 0 & $6 \% \mathrm{~b}$ & 0 & 0 & $33 \%$ & 0 \\
\hline Psychiatric disturbances $^{c}$ & $20 \%$ & $22 \%$ & $50 \%$ & $4 \%$ & $17 \%$ & 0 \\
\hline $\begin{array}{l}\text { Intentional/postural } \\
\text { tremor }\end{array}$ & \multicolumn{5}{|c|}{ tremor } & 0 \\
\hline Head titubation & 0 & $11 \%$ & 0 & $4 \%$ & $17 \%$ & 0 \\
\hline Choreiform movements & $27 \%$ & $17 \%$ & 0 & 0 & 0 & $100 \%$ \\
\hline \multicolumn{7}{|l|}{$\downarrow$ Sensory response: } \\
\hline Deep & $7 \%$ & $11 \%$ & 0 & $12 \%$ & $17 \%$ & 0 \\
\hline Skin & $20 \%$ & $17 \%$ & $6 \%$ & $8 \%$ & 0 & 0 \\
\hline Hyporreflexia $^{d}$ & 0 & $44 \%$ & $44 \%$ & $8 \%$ & $17 \%$ & $50 \%$ \\
\hline Hyperreflexia & $47 \%$ & $17 \%$ & $13 \%$ & $44 \%$ & 0 & 0 \\
\hline$\downarrow$ Muscular strength & $27 \%$ & $33 \%$ & $25 \%$ & $16 \%$ & $17 \%$ & $50 \%$ \\
\hline$\downarrow$ Abdominal skin reflexes & $13 \%$ & $22 \%$ & $44 \%$ & $28 \%$ & $17 \%$ & $50 \%$ \\
\hline
\end{tabular}

$n=$ number of examined patients.

IDiminished/absent.
aLoss of visual acuity, macular dystrophy, cone-rods dystrophy, photoreceptors disturbance.

bCorrelated with repeat number.

Insomnia, depression, personality changes (aggressiveness), cognitive deficit.

dOsteotendinous.

2 years of age; a mother with a 44 CAG allele transmitted a 64 CAG allele with disease onset at age 6 years; and a 39 CAG maternal allele expanded to 49 CAG produced a disease onset at age 8 years. A fourth case in a boy (at age 9 years), whose parents genotypes were unknown, had a 50 CAG allele.

Most cases had a disease onset in the third decade of life (mean age $=27.41$ (range 15-36 years of age, mode $=32$ ), $n=17$ adult cases). The most frequent first symptoms were loss of equilibrium (75\%) and gait disturbances (75\%).

A highly inverse correlation between CAG size and age of onset was observed, with $\mathrm{R}^{2}=0.8238$ and $\mathrm{y}=-0.0385 \mathrm{x}+2.9844$ for a log-transformed age of onset ( $n=19$ observations).

Heterozygosity of the CAG repeats in the normal range was low $(\mathrm{h}=0.55)$, allele 22 being the most frequent $(64.5 \%)$, followed by allele $21(17.1 \%)$.

There was no geographic aggregation of families with SCA2; 13 had remote ancestors born in Venezuela, scattered from occidental to oriental states along $800 \mathrm{~km}$ (Falcón to Bolívar) (Figure 1), which did not suggest a founder effect, and thus, haplotype analysis was not performed; one index case including convulsions with infantile disease onset, came from Marahuaca (Amazonas State), and he had Yanomami (isolated Amerindian) immediate ancestors; one family came from Perú (Ica).

\section{SCA3}

A total of 225 individuals at risk of being SCA3 carriers (both affected and asymptomatic) were assessed for expanded alleles at the ATXN3 locus (14q32.12), of which 41 had expansions, with allele sizes between 51 and 119 CAG. Meiotic instability of the mutated alleles in maternal transmissions was lower than that of paternal transmissions; the most frequent change in the former group was +2CAG (range -14 to +2 ), while in paternal transmissions, the range was much wider $(-10$ to +39$)$. Age of symptoms onset showed a weak non-significant correlation with the number of CAG repeats, with $\mathrm{R}^{2}=0.0952, \mathrm{y}=-0.007 \times+2.0721$ for a log-transformed age of onset ( $n=24$ observations). Disease onset frequently occurred in the fourth decade of life (mean age: 39 years (range 14-60 years, mode $=38$ )), and the frequent first symptom was loss of equilibrium (62.5\%).

The normal range of CAG repeats was very polymorphic, with 16 different alleles between 17 and 35 CAG; the most frequent allele was 26 CAG (23.8\%), followed by 17 CAG (15.5\%) and 18 CAG (13.1\%). Heterozygosity was $\mathrm{h}=0.888$.

SCA3 did not show geographic aggregation in the country, thus, haplotype analysis was not performed, although many independent families $(n=8)$ came from Falcón State (Figure 1$) ; 14$ out of 16 families had remote Venezuelan ancestors; the two remaining families came from Chile (Valparaíso) and Perú (Cerro de Pasco).

\section{SCA7}

Normal allele range varied between 9 and 12 CAGs, allele 10 being the most frequent (43.8\%), followed by allele 11 (25\%); heterozygosity value was $\mathrm{h}=0.76$.

This subtype had also the largest number of affected individuals in the sample. Fifty-six out of 112 individuals at risk had an expanded 


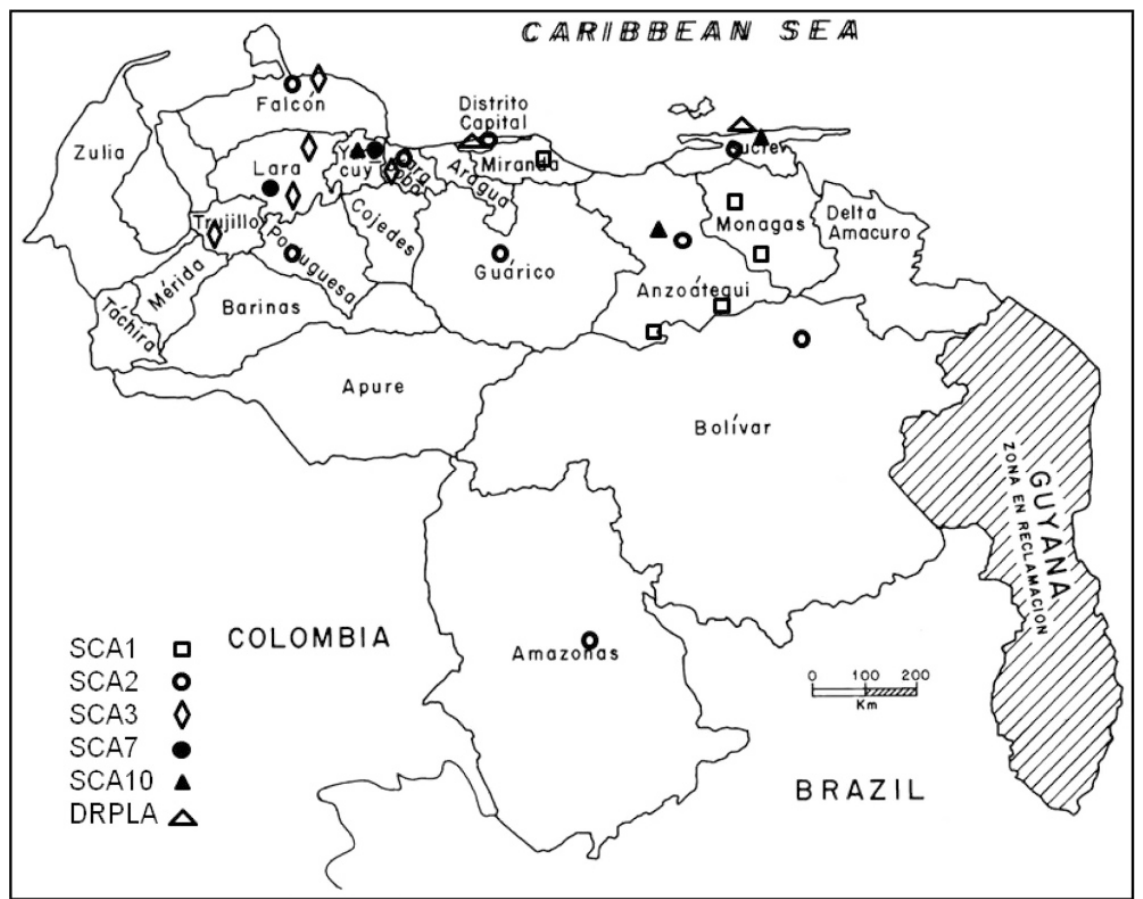

Figure 1 Geographic distribution of frequent SCA subtypes in Venezuela. In SCA1, only geographic foci with haplotype sharing (45\% of the families) are shown. For SCA7, two foci are shown (EI Tocuyo, Lara state and Nirgua, Yaracuy state), from which $76.5 \%$ of families ancestors come. In SCA10, families ancestors were $750 \mathrm{~km}$ afar, but all had the same Amerindian in-phase haplotype. SCA2 families were scattered across the country, and SCA3 families were most frequent in the western states of Falcón and Lara. Only two independent families showed DRPLA.

allele at the ATXN7 locus (3p14.1), between 35 and 58 CAG. Disease onset occurred mostly in the third and in the fourth decade of life ( mean age $=30.6$ years (range $8-60$ years, mode $=41$ years), $n=28$ cases). The most common first symptom was visual impairment $(70.6 \%)$, manifested as loss of visual acuity, frequently in the childhood and the adolescence (between 8 and 20 years of age). Ophthalmological findings before the complete loss of vision included diplopia, macular dystrophy and cone-rods dystrophy. The ophthalmic report was not available in all the cases.

Meiotic instability of the mutated alleles was noteworthy greater in paternal transmissions; none of the 15 male meiosis had allele size contractions, and the average of variation in CAG repeats was 9.1 (range 0 to $+20 \mathrm{CAG}$ ); in 11 female meiosis, the average was 3.3 CAG (range -6 to $+18 \mathrm{CAG}$ ) and only one maternal transmission had a large expansion of +18 CAG.

Age of disease onset showed a high correlation with CAG number of repeats $\mathrm{R}^{2}=0.6819 ; \mathrm{y}=-0.0287 \mathrm{x}+2.6977$ (log-transformed age of onset; $n=28$ cases with known age of onset).

All the studied families had remote ancestors born in Venezuela. There was a strong geographic aggregation into two foci ${ }^{23}$ for most families. Remote ancestors of nine independent families came from a geographic focus with center in Nirgua, Yaracuy state, while four other independent families had remote ancestors from Lara state, with focus center in El Tocuyo (Figure 1). Haplotype analysis revealed two likely independent founder effects in two foci. Haplotype 2A;2TG;4T was in phase with the expanded allele in eight out of nine independent index cases at the Nirgua focus; one of the families was partially uninformative, as the in-phase haplotype could not be established (as there were no available relatives); nevertheless, the index case genotype (2A/3A;1TG/2TG;3T/4T) strongly suggests a similar in-phase haplotype, exclusive of that geographic focus. At the El Tocuyo focus, haplotype 3A;2TG;4T was in phase in three out of four independent
Table 2 Frequency distribution of ATXN7 haplotypes in control and mutated chromosomes

\begin{tabular}{lccc}
\hline Haplotype & $\begin{array}{c}\text { Controls } \\
(\mathrm{n}=36) \%\end{array}$ & $\begin{array}{c}\text { In-phase } \\
(\mathrm{n}=14) \%\end{array}$ & $\begin{array}{c}\text { Geographic origins of index } \\
\text { cases remote ancestors }\end{array}$ \\
\hline 2A;2TG;4T & 41.7 & 64.3 & Nirgua, Yaracuy \\
3A;2TG;4T & 19.4 & 28.6 & El Tocuyo, Lara \\
3A;1TG;4T & 2.8 & 0.0 & Ospino, Portuguesa \\
3A;2TG;3T & 8.3 & 7.1 & \\
3A;1TG;3T & 2.8 & 0.0 & \\
2A;2TG;3T & 13.9 & 0.0 & \\
2A;1TG;3T & 2.8 & 0.0 & \\
\hline
\end{tabular}

$n=$ chromosomes.

INDEL order in haplotypes: rs144754108 (insA); rs3841968 (delTG); rs3836530 (delT).

families; in the fourth family, as in the previous case, index case genotype (2A/3A;1TG/2TG;3T/4T) might suggest a haplotype sharing with the other three families.

Table 2 shows the frequency distribution of haplotypes in control and in mutated chromosomes. In a control population sample coming from the same geographic areas of the SCA7 families, a great diversity was observed, with eight different haplotypes, while in the carriers and affected individuals, only three haplotypes were in-phase. The two most common haplotypes in the controls were also so in the carriers, suggesting that the founder mutation in each focus arose in a population with the frequent genetic background.

Allelic frequencies of each polymorphic marker compared with other worldwide populations are shown in Table 3; observed heterozygosity (h) values in controls ( $n=32$ chromosomes) were $\mathrm{h}=0.47$ (rs144754108), $\mathrm{h}=0.22$ (rs3841968) and $\mathrm{h}=0.45 \quad$ (rs3836530). 
Venezuelan allelic frequencies of the three INDELs were closer to those from the Iberian populations, which are one of the main ancestral populations originating our genetically admixed inhabitants.

\section{SCA10}

ATXN10 pentanucleotide repeats (ATTCT) were found to be highly polymorphic and stable in parental transmissions within the normal range. In a sample of 1576 normal chromosomes, heterozygosity was $\mathrm{h}=0.821$, with 15 different alleles. A total of 101 individuals at risk were ascertained for expanded alleles at ATXN10 (22q13.31). Three independent families had large expansions, with eight carriers (symptomatic and asymptomatic). Symptomatic patients with known age of onset $(n=3)$ initiated after the third decade of life (between 33 and 46 years, mode $=33$ ).

There was no geographic aggregation; origins of the remote ancestors of the index cases were $750 \mathrm{~km}$ far apart, from eastern to western states in the country (Carúpano, Sucre state to Nirgua, Yaracuy state) (Figure 1). Intragenic polymorphic markers analysis revealed the unique in-phase haplotype C;A;G;G;C;C (rs5764850; rs136003; rs72556348; rs72556349; rs72556350; rs136005) in the three SCA10-detected families.

\section{DRPLA}

Two independent individuals out of 168 had a CAG expansion at the ATN1 locus (12p13.31), with age of onset at 28 and 61 years, respectively. The most outstanding initial sign in them was choreiform movements. Both index cases had Venezuelan remote ancestors, from Caracas and probably from Cumaná (Sucre state), respectively (Figure 1). CAG repeats at ATN1 were highly polymorphic in the normal range (5-24 CAG), with 18 different alleles and a heterozygosity $\mathrm{h}=0.886$ ( $n=462$ chromosomes).

\section{DISCUSSION}

As dominant SCAs are a group of diseases with genetic heterogeneity, a wide variety of molecular pathogenesis is possible. Ataxia is the prominent sign, but many other complex symptoms accompany the phenotype, producing an overlap of clinical manifestations between the different subtypes. In addition, a large variability within family members can be observed. Thus, a correct diagnosis and classification is not possible without directly studying the possible altered genes. This approach has resulted in information about differences in regional frequencies of subtypes of SCAs worldwide, which is of great value for diagnosis and for establishing eventual genetic epidemiological registries.
In Venezuela, figures for SCA frequencies had not been established. To date, the Laboratory of Human Genetics at IVIC is the single center in the country in which a molecular diagnosis for these diseases can be made; thus, the studied sample is integrated by families from the country at large. A general prevalence of 1:25000 nuclear families during the 1990-2015 period can be estimated, a figure that still might be an underestimation because of an incomplete detection. The phenotype usually has a late onset and therefore might show additionally in some cases an apparently low penetrance explained by the mutation instability in the number of repeats. The observed prevalence is, however, quite similar to that referred to in a recent report of worldwide prevalence studies, in which the range was $1.5-4.0 \times 10^{5}$ (average $\left.2.7 \times 10^{5}\right) .^{3}$

In the Venezuelan families with clinical diagnosis of a dominant ataxia, molecular search among the most frequent causative genes worldwide was undertaken. A proportion of $43 \%$ of families remained with unidentified subtypes. As has been quoted from different populations, the range in the number of families without molecular diagnosis after systematic testing, goes from 33 to $92 \%$, with a mean of $44 \% .3,24,25$

In the $57 \%$ of families with identified genes, the frequency distribution of subtypes was different from that usually found in many populations. SCA3 is the most common subtype worldwide, followed by SCA1, SCA2, SCA6 and SCA7. ${ }^{1,3}$ In Venezuela, SCA7 was the most frequent one, with also the highest number of affected subjects, followed by SCA3, SCA2, SCA1, SCA10 and DRPLA, but SCA6, SCA8 and SCA17 were absent among 115 families.

The same clinical manifestations in the identified SCAs were frequently found in all subtypes, but some specific features were typical of a particular ataxia. Nystagmus was the most frequent oculomotor alteration in SCA3 patients $(81 \%)$, as has been reported in Cuban patients, ${ }^{26}$ as well as psychiatric manifestations. Intentional and/or postural tremor was frequent in SCA1 (40\%), and hyperreflexia was common to SCA1 and SCA7. The number of CAG repeats was a good predictor of the age of onset for SCA1, SCA2 and SCA7, and explained $58 \%, 82 \%$ and $68 \%$ of the variance, respectively, but it was not so for SCA3, in which the age of onset was poorly correlated with the length of CAG expanded alleles $\left(R^{2}=0.095\right)$, as has been quoted. $^{27}$

SCA3 (Machado-Joseph disease) is very frequent in Brazil $(30-92 \%)^{28}$ and Portugal $(57.8 \%),{ }^{29}$ as well as in some Asian populations: Japanese (Nagano and Niigata, 34-43.1\%), ${ }^{30}$ Chinese mainland, southeastern and Taiwan (34.7-72.5\%) and Singaporeans $(41.7 \%)$. It is infrequent in northern India $(3 \%)^{31}$ and Italy at large $(1 \%) .^{32}$ It has been proposed that the present worldwide frequency distribution of the disease is a result of a founder effect into Portugal

Table 3 Population allele frequencies of ATXN7 INDELs used to construct haplotypes

\begin{tabular}{|c|c|c|c|c|c|c|c|}
\hline \multirow{2}{*}{$\begin{array}{l}\text { INDEL } \\
\text { Allele }\end{array}$} & \multicolumn{7}{|c|}{ Population } \\
\hline & $A F R \% \mathrm{n}=176$ & $E U R \% \mathrm{n}=758$ & $A S N \% \mathrm{n}=572$ & $I B S \% \mathrm{n}=28$ & $C L M \% \mathrm{n}=120$ & $M X L \% \mathrm{n}=132$ & $V Z L \% n=32$ \\
\hline rs144754108 insA (3A) & 8.5 & 16.0 & 17.0 & 28.6 & 18.0 & 23.0 & 37.5 \\
\hline$-(2 A)$ & 91.5 & 84.0 & 83.0 & 71.4 & 82.0 & 77.0 & 62.5 \\
\hline rs3841968 delTG (1TG) & 30.7 & 39.0 & 14.0 & 3.6 & 11.0 & 6.0 & 12.5 \\
\hline$-(2 \mathrm{TG})$ & 69.3 & 61.0 & 86.0 & 96.4 & 89.0 & 94.0 & 87.5 \\
\hline rs3836530 delT (3T) & 26.0 & 18.0 & 17.0 & 25.0 & 22.0 & 25.0 & 34.3 \\
\hline$-(4 \mathrm{~T})$ & 74.0 & 82.0 & 83.0 & 75.0 & 78.0 & 75.0 & 65.6 \\
\hline
\end{tabular}

Abbreviations: AFR, Africans from Yoruba, Nigeria; ASN, East Asians; CLM, Colombians from Medellín; EUR, European Caucasoids; IBS, Iberians from Spain; MXL, Mexicans; VZL, Venezuelans. $n=$ \#chromosomes.

Population allelic frequencies quoted from 1000 genomes Project, except VZL. 
(mainland) from the Azores islands, where the original Machado and Joseph families dwelled, and later, it extended from Portugal to South Asia, perhaps through the heavy Portuguese maritime trade along past centuries. This hypothesis has been supported by a haplotype analysis performed in 249 families of 16 different countries from four continents, which shared the same intragenic A;C;A haplotype present also in the island of Flores (Azores), suggesting a very old mutational event and a founder effect in the different countries. ${ }^{33}$ In Venezuela, SCA3 had a frequency of $25.0 \%$, with 16 independent families spread out in the country. As there was no strong geographic aggregation, a regional founder effect seems unlikely. The Portuguese are not major direct contributors to Venezuelan old admixed populations; thus, the relatively high frequency of SCA3 families in the country could be attributed to more recent (nineteenth century) Portuguese immigrants or even more likely indirectly through Canarian immigrants with remote Portuguese ancestors. On the other hand, it is interesting to note that the most frequent allele in the control chromosomes was a large normal one (26 CAG); a long normal CAG allele at the ATXN3 has been correlated with higher frequencies of SCA3 in Japanese, compared with Caucasoid ${ }^{34}$ suggesting a mutation-prone situation.

Contrary to SCA3, SCA7 showed strong geographic aggregation. Remote ancestors of 13 out of 16 independent families came from two different geographic foci in Yaracuy state (focus center in Nirgua) with 9 families in a region with historically early abundant African slaves imported around 1550 to work in the newly discovered gold mine of Buría $^{35}$ and with 5 families in Lara state (focus center in El Tocuyo, in which only Caucasoid founders were present since 1545).

Haplotype analysis revealed a unique intrafocus in-phase haplotype in all the families in each case, but different ones between foci (Table 2). This finding suggests two independent founder effects for almost all SCA7-detected families in Venezuela. SCA7 is infrequent in most populations worldwide $(<6 \%)$; however, founder effects have been identified in some countries. In Scandinavia, the most frequent subtype in Sweden and Finland is SCA7, and all the affected families share a common in-phase haplotype covering $1.9 \mathrm{cM}$ (two flanking microsatellites D3S1287 and D3S1228, and the intragenic SNP rs3774729), ${ }^{36}$ supporting a strong founder effect in that region. In South Africa, a founder effect in families of African ethnic origin has been found. ${ }^{37}$ Similarly in Mexico, a very high prevalence of SCA7 was found in Veracruz (85.9\%), and a founder effect could be established through haplotype analysis with four flanking microsatellites and one intragenic SNP (D3S3566-D3S3698-D3S1600-(CAG) $)_{n}$-rs3774729D3S1287). A common in-phase haplotype was shared by $96.7 \%$ of SCA7 carriers from those 18 families; ${ }^{38}$ in addition, the ethnic origin of the chromosomal region containing the ATXN7 mutation was assessed and found to be of European ancestry.

Intragenic INDELs used to construct haplotypes in the Venezuelan individuals spanned a $108-\mathrm{Kb}$ region, from intron 1 to $3^{\prime} \mathrm{UTR}$ at the ATXN7 locus; thus, the different allelic disequilibrium in the two in-phase haplotypes strongly suggests two independent origins for the SCA7 mutation in both geographic foci. A third haplotype carried by one family coming from a different geographic region indicates an alternate origin than those in the other foci. A remote ancestral ethnic source for the mutation in each geographic focus could not be established with certainty; but allelic frequencies of INDELs in the general population were closer to those in the Iberian populations (Table 3), which were important contributors during the colonial period (sixteenth to eighteenth centuries) and later on to our admixed inhabitants. Furthermore, the 3A allele (rs144754108) carried by the El Tocuyo focus haplotype is more frequent in the Iberians than in
Africans, whereas the alternate allele 2A carried by the Nirgua focus haplotype is largely more frequent in Africans and therefore that might be a likelier ethnic origin for the mutation in that focus (Table 3). Additional studies are needed to assess the probable remote ethnic origins in those families.

In SCA10 families, another feature was observed. The three identified independent index cases had remote ancestors coming from three far apart states in the country with maximal afar distance of $750 \mathrm{~km}$ and a minimal of $338 \mathrm{~km}$ (Carúpano, Sucre state, Aragua de Barcelona, Anzoátegui state and Nirgua, Yaracuy state) (Figure 1). This wide geographic distribution did not suggest a unique founder effect for the disease in the country, but the intragenic polymorphic markers analysis revealed the same in-phase haplotype $C ; A ; G ; G ; C ; C$ (rs5764850; rs72556348; rs72556349; rs72556350; rs136005) in the three families.

This ataxia subtype has been found only in Amerindian Iberoamerican populations, mainly from Mexico (13.9\%) and Brazil $(0.7-3.0 \%),{ }^{28}$ and recently in a patient with a Sioux native of North American descent. ${ }^{39}$ The in-phase haplotype found in the Venezuelan families was indeed the same reported in Mexican and Brazilian ones and recently also in the Sioux patient, supporting a single and very remote common origin. Furthermore, two out of the three Venezuelan index cases carried the Amerindian allele 9 of D9S1120.

These findings strongly suggest an old Amerindian origin for the disease, probably in North American populations, from where it might have extended southward to Central and South America. Lakota-Sioux Amerindians have been found to be related to Meso and South American Amerindians, according to HLA haplotypes. ${ }^{40}$

It has been reported that the Mexican SCA10 patients phenotype is associated with seizures, supposedly in contrast to the Brazilian phenotype. Venezuelan patients had both phenotypes and the same remote ethnic descent; two out of the three families had seizures. In the family without convulsions, there were six symptomatic adult members with no record of seizures.

SCA2, the third most frequent ataxia subtype in the Venezuelan patients is common to almost all countries, with prevalence varying between 6 and $86.8 \% .{ }^{28}$ In some populations, its frequency is high, as in Cantabria, Spain (33\%) and Padua, Italy $(45 \%),{ }^{3}$ but the highest prevalence worldwide (41:100 000 inhabitants, $86.8 \%$ of patients with SCAs) has been reported in the Province of Holguín, Cuba, ${ }^{4}$ where a founder effect of Spanish origin from the seventeenth century was proposed. ${ }^{41}$

The frequency of Venezuelan families with SCA2 (21.9\%) is relatively high, compared with the general worldwide prevalence (15\%). ${ }^{1}$ Nevertheless, the geographic distribution of remote ancestors of index cases did not suggest a founder effect of the disease in the country, as they were scattered in 10 different states, from western to eastern and northern to southern regions across almost $800 \mathrm{~km}$ in both directions. Among all studied ataxias, SCA2 had the greatest variability in allele sizes through transmissions, of both maternal and paternal meiosis. Within 25 meiosis, only 7 (28\%) had the expanded allele size unchanged.

The CAG number of repeats was a very good predictor for the age of onset, and explained $>80 \%$ of the observed variability $\left(\mathrm{R}^{2}=0.8238\right)$, as mentioned. An interesting observation was the ascertainment of an infantile patient with a supposedly pure Amerindian ancestry. He came from a very isolated selvatic community of Yanomami (Carib) Amerindians; his genealogical links are still unknown to us. A confident alternate Amerindian source, as either an inherited or a sporadic ATXN2 change, could not be established for a mutation which has had so far a predominant Caucasoid origin. $\mathrm{He}$ 
presented with a confounding sign, because according to his ethnic origin, the seizures he presented should pinpoint to a diagnosis of SCA10, but surprisingly he carried an ATXN2 mutation with a high number of repeats (50 CAG).

SCA1 is present in almost all worldwide populations, with varying frequencies between 6 and 34\% depending on the geographic area. ${ }^{42}$ With few exceptions, ${ }^{43}$ no founder effects have been reported in most populations, although a higher prevalence in Caucasoids compared with Asians has been noticed. ${ }^{34}$ In Venezuela, among patients with dominant cerebellar ataxia, it is the fourth most frequent subtype (17.2\%). It shows geographic aggregation in southern Anzoátegui, northwestern Monagas and northeastern Bolívar states along the north and south Orinoco river shores (Figure 1). A very old ancestral common origin hypothesis for this mutation was supported by the same in-phase haplotype insTTTAA;G;C shared by the families coming from that extensive area ( $300 \mathrm{~km}$ long), which was absent in control and other SCA1 families chromosomes. Ethnic origin of the ancestral mutated chromosome, however, could not be clearly established, although in-phase alleles insTTTAA, G and C are highly prevalent in African Sub-Saharan populations (0.79, 0.66 and 0.91, respectively).

DRPLA is a very infrequent ataxia subtype worldwide, being absent from many populations (Australia, Cuba, Finland, Brazil and European countries). ${ }^{28,44}$ It is, however, very frequent in Japan (19.8\%) and Portugal (11.2\%), where a founder effect has been proven with the finding of a common haplotype shared by mutated chromosomes in Japanese and Portuguese patients, which besides is also very frequent in Japanese controls, but rare in Portuguese control chromosomes. ${ }^{45}$ In the Venezuelan patients with clinical diagnosis of a dominant ataxia, two independent index cases were found (3.1\%). None of the families had recent Portuguese or Japanese ancestors; all were of remote Venezuelan ancestry.

DRPLA might be searched sometimes for the differential diagnosis of Huntington disease, when similar clinical manifestations, as chorea and dementia are present. ${ }^{11}$

The genetic epidemiological data of dominant SCAs gathered during the last 30 years in Venezuela are a very useful resource for diagnosis and genetic counseling of Venezuelan families. Knowledge of the geographic location of the different subtypes in the country as well as of clinical differences among SCAs eases the molecular search in new families. The observed frequency of mutations suggests that for families without visual impairment, search should be tried first for SCA3 and SCA2 genotypes, followed by SCA1, SCA10 and DRPLA. For those without these subtypes, the remaining ataxias have to be tested. Caucasoid, African and lastly Amerindian sources in this order are likely origins for the most frequently found types of dominant SCAs in Venezuela as discussed.

\section{CONFLICT OF INTEREST}

The authors declare no conflict of interest.

\section{ACKNOWLEDGEMENTS}

To the physicians who referred us many of the patients, especially Dr Gisela Ramírez and Dr Roberto Weiser. To Alba Marina Florez for her efficient processing of the database. To Mary Acosta for her assistance with the capillary electrophoresis standardization. To Yuri Yépez for his standardization of the SCA3-RP protocol.
1 Bird, T. D. Hereditary Ataxia Overview, GeneReviews [Internet], 28 Oct 1998 (updated 5 Mar 2015) (eds Pagon, R. A., Adam, M. P., Ardinger, H. H. et al.) (University of Washington, Seattle, WA, 1993-2015). Available from: http:/www.ncbi.nlm.nih.gov/ books/NBK1138/ (accessed April 2015).

2 Rüb, U., Schöls, L., Paulson, H., Auburger, G., Kermer, P., Jen, J. C. et al. Clinical features, neurogenetics and neuropathology of the polyglutamine spinocerebellar ataxias type 1, 2, 3, 6 and 7. Prog. Neurobiol. 104, 38-66 (2013).

3 Ruano, L, Melo, C, Silva, M. C. \& Coutinho, P. The global epidemiology of hereditary ataxia and spastic paraplegia: a systematic review of prevalence studies. Neuroepidemiology 42, 174-183 (2014).

4 Orozco, G., Estrada, R., Perry, T. L., Arana, J., Fernández, R., González-Quevedo, A. et al. Dominantly inherited olivopontocerebellar atrophy from eastern Cuba: clinical, neuropathological, and biochemical findings. J. Neurol. Sci. 93, 37-50 (1989).

5 Jonasson, J., Juvonen, V., Sistonen, P., Ignatius, J., Johansson, D., Björck, E. J. et al. Evidence for a common Spinocerebellar ataxia type 7 (SCA7) founder mutation in Scandinavia. Eur. J. Hum. Genet. 8, 918-922 (2000).

6 Rasmussen, A., Matsuura, T., Ruano, L., Yescas, P., Ochoa, A., Ashizawa, T. et al. Clinical and genetic analysis of four Mexican families with spinocerebellar ataxia type 10. Ann. Neurol. 50, 234-239 (2001).

7 Trott, A., Jardim, L. B., Ludwig, H. T., Saute, J. A. M., Artigalas, O., Kieling, C. et al. Spinocerebellar ataxias in 114 Brazilian families: clinical and molecular findings. (Letter). Clin. Genet. 70, 173-176 (2006).

8 Ikeda, Y., Ohta, Y., Kobayashi, H., Okamoto, M., Takamatsu, K., Ota, T. et al. Clinical features of SCA36: a novel spinocerebellar ataxia with motor neuron involvement (Asidan). Neurology 79, 333-341 (2012).

9 Garcia-Murias, M., Quintans, B., Arias, M., Seixas, A., Cacheiro, P., Tarrio, R. et al. 'Costa da Morte' ataxia is spinocerebellar ataxia 36: clinical and genetic characterization. Brain 135, 1423-1435 (2012).

10 Obayashi, M., Stevanin, G., Synofzik, M., Monin, M. L., Duyckaerts, C., Sato, N. et al. Spinocerebellar ataxia type 36 exists in diverse populations and can be caused by a short hexanucleotide GGCCTG repeat expansion. J. Neurol. Neurosurg. Psychiatry 86, 986-995 (2015).

11 Paradisi, I., Ikonomu, V. \& Arias, S. Huntington disease-like 2 (HDL2) in Venezuela: frequency and ethnic origin. J. Hum. Genet. 58, 3-6 (2013).

12 Orr, H., Chung, M., Banfi, S., Kwiatkowski, T., Servadio, A., Beaudet, A. et al. Expansion of an unstable trinucleotide CAG repeat in spinocerebellar ataxia type 1. Nat. Genet. 4, 221-225 (1993).

13 Zhuchenco, O., Bailey, J., Bonnen, P., Ashizawa, T., Stockton, D. W., Amos, C. et al. Autosomal dominant cerebellar ataxia (SCA6) associated with small polyglutamine expansions in the alpha 1A-voltage-dependent calcium channel. Nat. Genet. 15 62-69 (1997).

14 David, G., Abbas, N., Stevanin, G., Dürr, A., Yvert, G., Cancel, G. et al. Cloning of the SCA7 gene reveals a highly unstable CAG repeat expansion. Nat. Genet. 17, 65-70 (1997).

15 Koob, M., Moseley, M., Schut, L. J., Benzow, K. A., Bird, T. D., Day, J. W. et al. An untranslated CTG expansion causes a novel form of spinocerebellar ataxia (SCA8). Nat. Genet. 26, 379-384 (1999).

16 Matsuura, T., Yamagata, T., Burgess, D., Rasmussen, A., Grewal, R., Watase, K. et al. Large expansion of the ATTCT pentanucleotide repeat in spinocerebellar ataxia type 10. Nat. Genet. 26, 191-194 (2000).

17 Paradisi, I. \& Arias, S. Marked geographic aggregation of acute intermittent porphyria families carrying mutation Q180X in Venezuelan populations, with description of further mutations. J. Inherit. Metab. Dis. 33 (Suppl 3), S455-S463 (2010).

18 Cagnoli, C., Michielotto, C., Matsuura, T., Ashizawa, T., Margolis, R., Holmes, S. et al. Detection of large pathogenic expansions in FRDA1, SCA10, and SCA12 genes using a simple fluorescent repeat-primed PCR assay. J. Mol. Diagn. 6 96-100 (2004).

19 Cagnoli, C., Stevanin, G., Michielotto, C., Gerbino Promis, G., Brussino, A., Pappi, P. et al. Large pathogenic expansions in the SCA2 and SCA7 genes can be detected by fluorescent repeat-primed polymerase chain reaction assay. J. Mol. Diagn. 8, 128-132 (2006).

20 Kawaguchi, Y., Okamoto, T., Taniwaki, M., Aizawa, M., Inoue, M., Katayama, S. et al. CAG expansions in a novel gene for Machado-Joseph disease at chromosome 14q32.1. Nat. Genet. 8, 221-227 (1994).

21 Almeida, T., Alonso, I., Martins, S., Ramos, E. M., Azevedo, L., Ohno, K. et al. Ancestra origin of the ATTCT repeat expansion in spinocerebellar ataxia type 10 (SCA10). PIOS ONE 4, e4553 (2009).

22 Paradisi, I., De Freitas, L. \& Arias, S. Most frequent mutation c.3402delC (p.Ala1135GInfsX13) among Wilson disease patients in Venezuela has a wide distribution and two old origins. Eur. J. Med. Genet. 58, 59-65 (2015).

23 Arias, S. in: New Horizons in Neonatal Screening, Int Congr Ser, Vol. 1041 (eds Farriaux, J. P. \& Dhondt, J. L.). 97-100 (Excerpta Med, Amsterdam, 1994).

24 Dürr, A. Autosomal dominant cerebellar ataxias: polyglutamine expansions and beyond. Lancet Neurol. 9, 885-894 (2010).

25 Sandford, E. \& Burmeister, M. Genes and genetic testing in hereditary ataxias. Genes 5 586-603 (2014).

26 González-Zaldívar, Y., Vázquez-Mojena, Y., Laffita-Mesa, J. M., Almaguer-Mederos, L. E., Rodríguez-Labrada, R., Sánchez-Cruz, G. et al. Epidemiological, clinical, and molecular characterization of Cuban families with spinocerebellar ataxia type 3/ Machado-Joseph disease. Cerebellum Ataxias 2, 1 (2015). 
27 van de Warrenburg, B. P., Hendriks, H., Dürr, A., van Zuijlen, M. C., Stevanin, G., Camuzart, A. et al. Age at onset variance analysis in spinocerebellar ataxias: a study in a Dutch-French cohort. Ann. Neurol. 57, 505-512 (2005).

28 Cintra, V. P., Lourenço, C. M., Marques, S. E., De Oliveira, L. M., Tumas, V. \& Marques W. Jr. Mutational screening of 320 Brazilian patients with autosomal dominant spinocerebellar ataxia. J. Neurol. Sci. 347, 375-379 (2014).

29 Vale, J., Bugalho, P., Silveira, I., Sequeiros, J., Guimarães, J. \& Coutinho, P. Autosomal dominant cerebellar ataxia: frequency analysis and clinical characterization of 45 families from Portugal. Eur. J. Neurol. 17, 124-128 (2010).

30 Gan, S. R., Shi, S. S., Wu, J. J., Wang, N., Zhao, G. X., Weng, S. T. et al. High frequency of Machado-Joseph disease identified in Southeastern Chinese kindreds with spinocerebellar ataxia. BMC Med. Genet. 11, 47 (2010).

31 Faruq, M., Scaria, V., Singh, L., Tyagi, S., Srivastava, A. K. \& Mukerii, M. SCA-LSVD: A repeat-oriented locus-specific variation database for genotype to phenotype correlations in spinocerebellar ataxias. Hum. Mutat. 30, 1037-1042 (2009).

32 Brusco, A., Gellera, C., Cagnoli, C., Saluto, A., Castuccci, A., Michielotto, C. et al. Molecular genetics of hereditary spinocerebellar ataxia: mutation analysis of spinocerebellar ataxia genes and CAG/CTG repeat expansion detection in 225 Italian families. Arch. Neurol. 61, 727-733 (2004).

33 Gaspar, C., Lopes-Cendes, I., Hayes, S., Goto, J., Arvidsson, K., Dias, A. et al. Ancestral origins of the Machado-Joseph disease mutation: a worldwide haplotype study. Am. J. Hum. Genet. 68, 523-528 (2001)

34 Takano, H., Cancel, G., Ikeuchi, T., Lorenzetti, D., Mawad, R., Stevanin, G. et al. Close associations between prevalences of dominantly inherited spinocerebellar ataxias with CAG-repeat expansions and frequencies of large normal CAG alleles in Japanese and Caucasian populations. Am. J. Hum. Genet. 63, 1060-1066 (1998).

35 de Aguado, P. Recopilación historial de Venezuela [1581]. Morón G. 2da. pt. Libros I-III, Tomo 1. Bibl Acad. Nac. Hist. 62, 1-365 (1963)

36 Jonasson, J., Juvonen, V., Sistonen, P., Ignatius, J., Johansson, D., Björck, E. et al Evidence for a common Spinocerebellar ataxia type 7 (SCA7) founder mutation in Scandinavia. Eur. J. Hum. Genet. 8, 918-922 (2000).
37 Greenberg, J., Solomon, G. A., Vorster, A. A., Heckmann, J. \& Bryer, A. Origin of the SCA7 gene mutation in South Africa: implications for molecular diagnostics. Clin. Genet. 70, 415-417 (2006).

38 García-Velázquez, L., Canizales-Quinteros, S., Romero-Hidalgo, S., Ochoa-Morales, A., Martínez-Ruano, L., Márquez-Luna, C. et al. Founder effect and ancestral origin of the spinocerebellar ataxia type 7 (SCA7) mutation in Mexican families. Neurogenetics 15, 13-17 (2014).

39 Bushara, K., Bower, M., Liu, J., McFarland, K. N., Landrian, I., Hutter, D. et al. Expansion of the spinocerebellar ataxia type 10 (SCA10) repeat in a patient with Sioux native American ancestry. PLOS ONE 8, e81342 (2013).

40 Arnaiz-Villena, A., Parga-Lozano, C., Moreno, E., Areces, C., Rey, D. \& Gómez-Prieto, P. The origin of Amerindians and the peopling of the Americas according to HLA genes: admixture with Asian and Pacific people. Curr. Genomics 11, 103-114 (2010).

41 Velázquez Pérez, L., Sánchez Cruz, G., Santos Falcón, N., Almaguer Mederos, L. E., Escalona Batallan, K., Rodríguez Labrada, R. et al. Molecular epidemiology of spinocerebellar ataxias in Cuba: Insights into SCA2 founder effect in Holguin. Neurosci. Lett. 454, 157-160 (2009).

42 Subramony, S. H. \& Ashizawa, T. in: Spinocerebellar Ataxia Type 1. GeneReviews ${ }^{\circledR}$ [Internet], 1 Oct 1998 (updated 3 Jul 2014) (eds Pagon, R. A., Adam, M. P., Ardinger, H. H. et al.) (University of Washington, Seattle, WA, 1993-2015). Available from: http://www.ncbi.nlm.nih.gov/books/NBK1184 (accessed 26 April 2015).

43 Pujana, M. A., Corral, J., Gratacòs, M., Combarros, O., Berciano, J., Genís, D. et al. Spinocerebellar ataxias in Spanish patients: genetic analysis of familial and sporadic cases. The Ataxia Study Group. Hum. Genet. 104, 516-522 (1999).

44 Le Ber, I., Camuzat, A., Castelnovo, G., Azulay, J. P., Genton, P., Gastaut, J. L. et al. Prevalence of dentatorubral-pallidoluysian atrophy in a large series of White patients with cerebellar ataxia. JAMA Neurol. 60, 1097-1099 (2003).

45 Martins, S., Matamá, T., Guimarães, L., Vale, J., Guimarães, J., Ramos, L. et al. Portuguese families with dentatorubropallidoluysian atrophy (DRPLA) share a common haplotype of Asian origin. Eur. J. Hum. Genet. 11, 808-881. 